\title{
Application of Sadpba on Meecs System
}

\author{
D. Vasumathi, P. Rajarajeswari, and A. RamamohanReddy
}

\begin{abstract}
The architecture of a software system is concerned with the top-level decomposition of the system into its main components. we have accessed the issues about the key point is how to link some requirements to some architecture decisions such as creation of components and connectors with special features, adoption of architectural styles and patterns upon which the design will be built up, and establishment of communication protocols through which the whole system can be integrated. We provide software architecture centered design process called SADPBA. (Requirements, architecture and system details). We present the MEECS (Mobile Embedded E-Commerce System) by which we perform the explorative research on applying agent into mobile e-commerce.
\end{abstract}

Index Terms-Software architecture, reuse, architectural design space and rules, software architecture centered design process, mobile embedded E-commerce system.

\section{INTRODUCTION}

Software architecture is the study of the large-scale structure and performance of software systems [Shaw 89]. Important aspects of a system's architecture include the division of functions among system modules, the means of communication between modules, and the representation of shared information. The architectural alternatives available to a system designer can be described and classified by constructing a design space. Within a design space, we can formulate design rules that indicate good and bad combinations of choices. Such rules can be used to select an appropriate system design based on functional requirements. A valuable contribution will be made if the rules can help a journeyman designer to make choices comparable to those that a master designer would make or even just help the journeyman to choose a reasonable design with no major errors. With sufficient experience, a set of such rules may become complete and reliable enough to serve as the basis for automated system design, but the rules can be of practical use long before that stage is reached .In this paper section 2 provides Software architecture centered design process is called SADPBA, overview of SADPBA, section 3 presents Trace mechanism in SADPBA. Section 4 presents Case study of MEECS and also provide application of SADPBA on MEECS.

Manuscript received May 5, 2012; revised June 27, 2012

D. Vasumathi is with Department of Computer Science and Engineering, JNTU Hyderabad, India

P. Rajarajeswari is with Department of computer science and Engineering, Madanapalle Institute of Technology and Science, Madanapalle, India (email:perepicse@gmail.com).

A. RamamohanReddy is with Department of Computer Science and Engineering, S.V.University, Tirupathi, India.

\section{SADPBA}

In this section, we provide our software architecture centered design process; called SADPBA (Software Analysis and Design Process Based on Architecture). This process uses the ideas of design space in the mobile-based collaboration platform. In the following, we give the introduction to its overview, design space application and track relationships in them. This process follows this pattern, which is the topic of the next section. The overview of SADPBA is shown in Fig.1. Intuitively, through the analysis of SH, Res, Act and Cons of actions next to each other, designers can judge the correctness of transfer between design spaces.

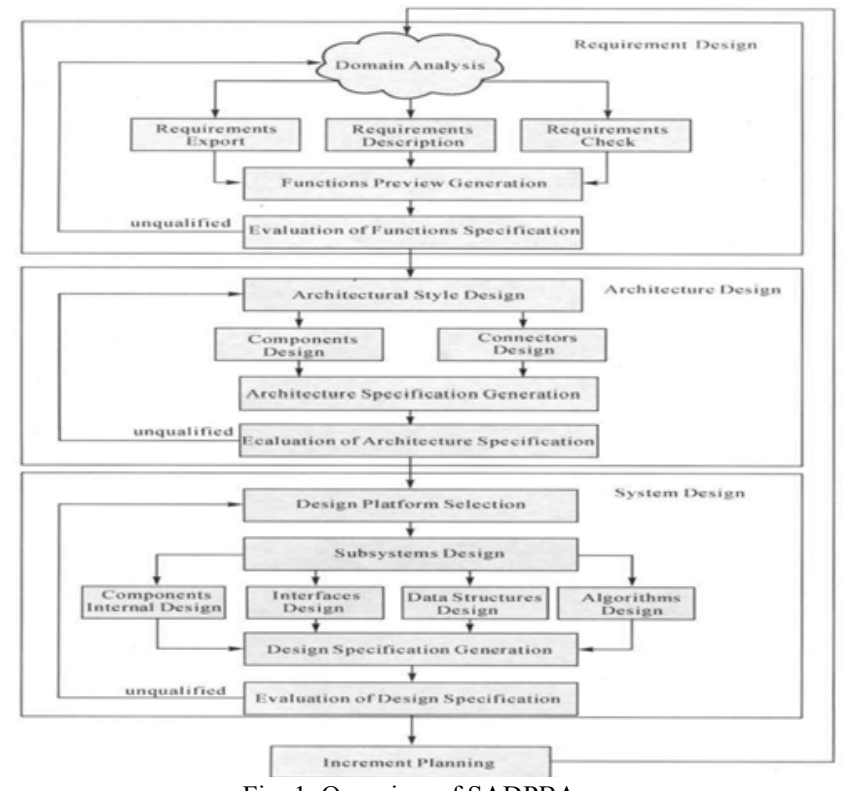

Fig. 1. Overview of SADPBA

And define the map relationships as: DS1 and DS2 are two design spaces (they may be the same one), if a rule exists, expressed as f, which makes any element $\alpha \in \mathrm{DS}_{1}$ has a counterpart $\beta$ in DS2, we say that $\mathrm{f}$ is a map from DS1 to DS2. This relationship is expressed as $\mathrm{f}=\mathrm{DS}_{1} \rightarrow \mathrm{DS}_{2}$ and $f(\alpha)=\beta$.

In this manner, it is possible to implement a "design machine" that is fed with requirements and give design results. This machine can also choose the best one, if we predefine an evaluation formula and assign weight to different dimensions. To achieve this point, we need gather the rules for mapping, normally in the way of comparing and summarizing the designs under design space specifications. Therefore, we own the capability of automatically judging whether a design is reasonable and potential pitfalls incurred 
by the hidden shortcomings.

\section{TRACE MEChANISM IN SADPBA}

For a tool capable of automatic design, it is important to judge whether its output is good or not. A validation achieves this, which calls for the information about mapping between every design spaces. This is why we need to extract the "trace" relationship in SADPBA.

Trace means a bidirectional relationship between two elements in one or multiple design spaces, which defined by a certain rules. More formally, In design spaces, if element $\alpha$ can be tracked to the element $\beta$, we say that element $\alpha$ and $\beta$ have traceable relationship, expressed as a Trace to $\beta$ (Since this relationship is bidirectional, given a Trace to $\beta$ Trace to a holds definitely.) In SADPBA, we categorize three kinds of traceable relationships. They come from the completeness of design spaces used in SADPBA. The first one stands between DSF and DSA: Given DSF is a Function Design Space and DSA is an Architecture Design Space, and $\mathrm{f}$ is a rule of map between them. Only when any element $\alpha \in \mathrm{DS}_{F}$ $\alpha$ and all its dependencies can be mapped to the element in DSA, DSv is complete to DSA. Another one exists between DSA and DSs: Given DSA is an Architecture Design Space and DSs is an System Design Space, and $\mathrm{f}$ is a rule of map between them. Only when any element $\alpha \in \mathrm{DS}_{\mathrm{A}}$ and all its dependencies can be mapped to the element in DSs, DSA is complete to DSs. Purely from mathematics, completeness means every element in a domain of a map can find a counterpart in its range. In design space, completeness guarantees that given an input in one design space we can find a result in the next design space for sure. The whole design process of SADPBA thus behaves determinably, which is the foundation of automatic design capable tool. SADPBA employs the sequence-based specification process. Each sequence indicates a use scenario. Through enumeration, permutation and combination of scenarios, SADPBA developers check and validate the design results and create deterministic traceable relationships.

- In the same space, elements that have dependency relationship have traceable relationship.

- When DSF is complete to DFA, the elements in DSF

- and in DSA have traceable relationship.

- When $D S_{A}$ is complete to DSs, the elements in DSA and in DSs have traceable relationship.

\section{STUdy CASE MEECS}

In this section, we present the Mobile Embedded E-Commerce System (MEECS), by which we perform the explorative research on applying agent into mobile ecommerce. In the development of this system, we introduce and refine SADPBA discussed above. In the following content, we first give an introduction to this system and then explain how SADPBA was used parts.

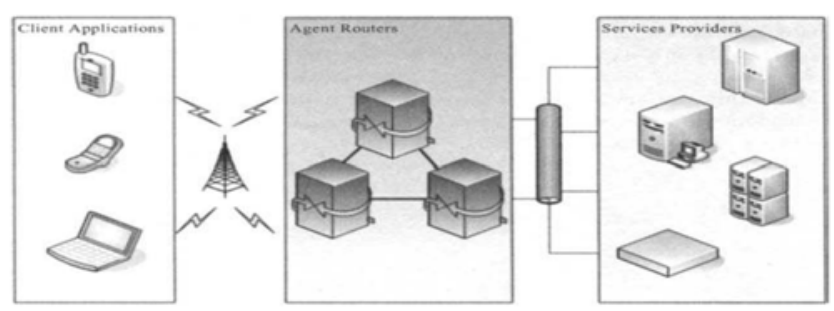

Fig. 2. Overview of MEECS

\section{A. Applying SADPBA in MEECS}

In this section, we briefly introduce the design process based on SADPBA, and describe how the decisions about architecture of MEECS are made by comprehensive consideration of requirements and other concerns such as techniques and research related. We start from the enumeration of primary requirements of our project. Just like what most people are doing nowadays, we use the artifact "use cases" document to record our project's goals. First, we categorize three kinds of actors in this system, the client users who use the handheld mobile devices, the administrators of Agent Router, and the vendors who provide services.

\section{B. Requirements Analysis}

The goal of client users is very simple: they should see the result generated by the service through user interface and can send requests to find and choose some services. The heterogeneity of display in various devices should be taken into account, which triggers our another research project, the Language facilitating Interface Representation under Limited Mobile Computing Environment (FIML) (Wang, 2003). Simply speaking, this is a markup language specifically for graphical display in different mobile devices. It is rather trivial to talk more about this language in this section. What you should know is that this language needs parsing and behaviors represented by it should be executed. Therefore, the use case diagram seems like what is shown in below Fig. 3 .

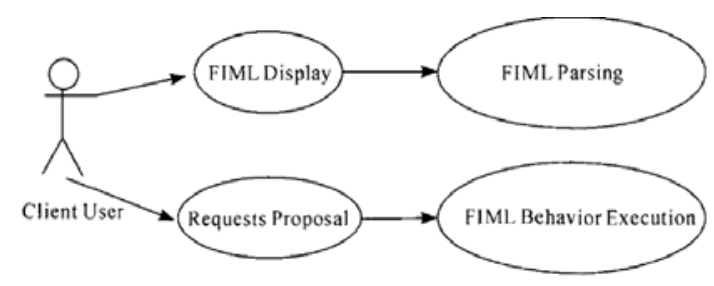

Fig. 3. Use case diagram of client user

The tasks of administrators are to maintain the Agent Router's map records, each of which bind a client user to a service. For this, we introduce the idea of administrator agent, an independent component capable of performing administrators' duties, such as registering or unregistering a client user, finding the suitable service brokers and finally clearing the channel enabling communication between client user and service directly. Other than the functional requirements, the performance and availability concerns need to find their solution here. In the architecture design of this part, you can see that how these requirements are met. The vendors have fewer responsibilities. For this system, they 
only need to implement their services by following a specification which guarantees that their services can be recognized and used by the agents. Since it is relative easy and not the key point of MEECS, we postpone its design until the late phase of system. Generalizing the points above, we generate three associated packages of use cases as the start point to the architecture design phase.

\section{Architecture Design}

In the architecture design phase, we separate the whole system into three sub-ones. The client, which is called as "terminal component" in the architecture design, focuses on the representation functions. And the Agent Router is designed as Mobile Embedded E-Commerce Platform (MEECP).

- Terminal: The architecture of terminal is shown in FigTerminal contains the components listed as following:

- Facade: Responsible of construct and display of user interface.

- Command: Parsing the behavior mark in the FIML interface, generating the command objects and then performing their execution.

- Parser: Parsing the mark of FIML and extracting the data embedded in them, preparing for further operations such as command execution or interface display.

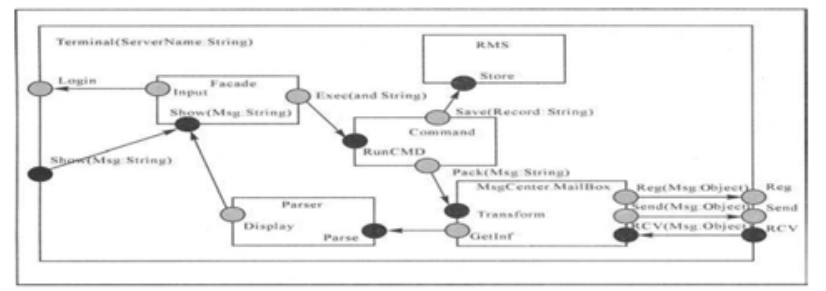

Fig. 4. Architecture of MEECS terminal

- RMS: Archiving the data permanently in the mobile terminal, enabling the operations of records query, insert, delete and-update. RMS (Record Management Storage) originally is the facility provided by J2ME for permanent record storage. We extend it to make convenient the operations of records designed in our own system.

- MsgCenter:. Under the mobile environment, network breaking off is the conventional problem. In this concern, MsgCenter is designed to work in the mail box style, or more canonical, the asynchronous transmission, allowing connection interrupt and resume. MsgCenter is the sole port that links the terminal distributed discretely in the net.The architecture for client supports three main processes: the generation of flexible user interface, the execution of behavior and the messages handling. The user interface is generated in the following steps. The FIML Parser component parses the control description in the markup language, and then extends the variables, replacing the configuration table, the system parameters and page parameters. The Facade component gets the parse result, a description of controls, and finally renders the display area. Execution of behaviors starts from the interaction from users. Once people trigger some behavior, its description is fed into the Command component, which first replaces the parameters in the description and extracts the elements of that behavior.
The command queue accepts the command and keeps it, until the command execution thread gets started and fetches this command for execution. In the process of behavior execution, if message should be sent to the MEECP, the MsgCenter component is activated. It maintains two queues, one for the received messages and another for the messages to be sent. To tackle this task,MEECP employs two threads respectively.

- MEECP:The MEECP component is responsible of service register, terminal register, handle of users' message and gives feedback. It has three ports: Reg, Send and $\mathrm{Rc}_{\mathrm{v}}$. Reg is the port enabling terminal's register and unregister. Each terminal which expects gain some services in MEECS should record its identifier and properties first, the latter of which is the basis to automatically reason about which services should be bound to them if more than one can be found and seem satisfactory. Send and $\mathrm{Rc}_{\mathrm{v}}$ are the ports for message communications. The register of services is finished by message manipulation. MEECP has five top level components, Bus, Super Server Agent, Administrator Agent, Broker Agent and Function Agent, where Bus is composed of MsgCenter (similar to the one in Terminal), Agent Management System (AMS) and Directory Facility (DF). The architecture of MEECP is shown in below Figure.

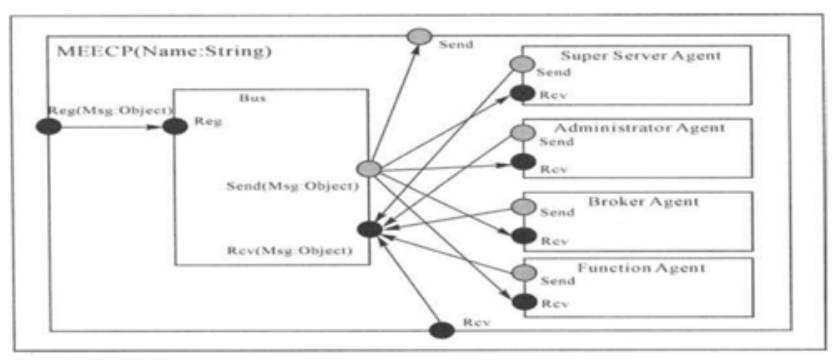

Fig. 5. Architecture of MEECP

To solve the problem of task allocation and the efficiency of communications among agents, MEECP introduces the Agent Layered Management Architecture. The agents are separated into five layers according to their duties. The Layer of Common Utility, supported by Agent Management System and Directory Function, facilitating the basic agent information keeping, including the identifier, status, time stamp and bound services. The messages coming from outside will be handled by this layer. Therefore, administrators of MEECP can impose policies by filtering out the messages needing to ignorance. The Layer of Management control all the agents deployed on the platform. And noticeably, it is itself also an agent, called Super Server Agent. In the perspective of functions, Super Server Agent does nothing about what the terminal wants, but is the start point of task allocation.

The Layer of Inception finishes the job of connecting one terminal to a carefully selected Function Agent, the proxy of actual services. In this layer, two kinds of agents, Administrator Agent and Server Agent cooperate to balance the load of numerous agents.

The Layer of Service Category is set for category of services. It is this layer that keeps the information of a set of 
related services registered and prepares to choose one for the terminal according to the guide tips attached by it in the form of properties.

The Layer of Domain Specific Services provides the final service proxy. After all steps of registering, the terminal and its bound services are linked by this kind of agents. Of course, one can realize the function in this agent so that there is no burden of service objects. However, doing this too much will extremely increase the load of MEECP and affects the response $\sim$ aeration to normal requests from other terminals.

The reason of creating such a pyramid like agents layers is to reduce the opportunities that some agents can afford the load while others stay leisure. In this architecture, terminal register in fact is a process of fetching different agent. On the one hand, the terminal can be implemented in a simple way in that it needs to know the other side of communication must be an agent (no matter which kind of agent, they have the unified communication protocols and identical access interface). On the other hand, MEECP guides the terminal to where they should go, that is, Super Server Agent chooses one Server Agent, Server Agent choose one Administrator Agent, Administrator Agent chooses one Broker Agent, and at last Broker Agent chooses one Function Agent. But why set five layers? Is all kinds of agents in the layer expect those of function only do the same job, that is, choose one agent in the next layer? This is not the case. Each kind of agents has duties of its own. Super Server Agent is created to allow the cluster of MEECP servers to compose a logic integrated whole, in which case several MEECP component instances can coexist and collaborate, wherever their physical location are. Similar for each MEECP server, agents are separated into multiple groups equipping with distinct administrative policies. And Broker Agent, as mentioned above, manages a collection of services that can fall in the same category, facilitating the implementation of match engines that attempt to follow terminals' requirements for service because different categories of services have different concepts and rules. The Agent Management System (AM S) is a framework in which various agents can be created, disposed, located, transferred and communicated to. With the help of AMS, agents are able to enter an agent group, search services provided in it or even connect with agents in other group or serve What's more, AMS controls the activation of agents. AMS will allocate a thread from a thread pool to execute code embedded in the agent and collect the taken resource if an agent's reference count reduces to zero. The Directory Function (DF) is a table maintaining the information about terminals, agents and services. Aside from the respective information of these three entities, DF records their bind relationship. For example, when a terminal finishes its register, the corresponding Super Server Agent, Server Agent, Administrator Agent, Broker Agent and Function Agent will be attached in the record. DF uses the transactions based on the database, to guarantee $t$ atomicity and consistency. 'System Design It is time to convert architecture to implementation-related models, where we use UML to sketch the system that will be coded in Java. Fig. 7 is the class enumeration diagram (relationships among classes are ignored here). Explaining every class is trivial and thus we do not want to rove in the detail of implementation with more broad statements. But we want to clarify the path from requirements to implementation. In a domain, the consideration should be emphasized on extracting and describing the domain specific concepts during the requirements analysis. Those concepts related to the solution of problem require much more attention. The result of this phase is so called domain model, which although cannot solve the problem but provide an order-of-magnitude sense to aid developers to gain a deeper insight.

In the case of MEECS, the concepts such as mobile client, agent, register information keeper, are identified to define the requirements. The requirement analysis is in fact an action that finding the constraints and conditions the entities in the domain model have to conform to. Note that the object model is neither a description of architecture nor class or objects in the Object-Oriented development, but only a simplification and visualization of concepts in the problem world. The architecture design is the first step getting approach to the solution in the high level of the system. Although algorithms are so important to succeed, they only deal with the problems about computation, which is only a small part in the usable software. More broadly, requirements of quality attribute, performance, usability, availability, security and so on, have to be tackled by the cooperation of decomposed elements. In the architecture design, concepts are separated according to the requirements into elements and regulate their interaction. In MEECP, for example, in order to balance the load, we design the layered agents. Abstractly, this is a map from function design space to the architecture design one, which if gotten verified again and again we can put in the rules of map created for this domain, and then contributes to the automatic design. Architecture design is programming language neutral in which only the overall sight of final system is generated. Finally in the system design, we determine the techniques and convert the architecture into the model that is easily to be coded.

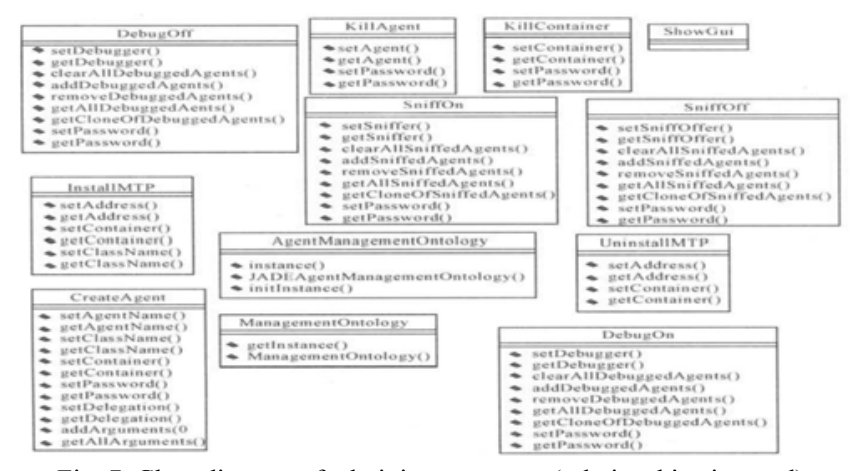

Fig. 7. Class diagram of administrator agent (relationships ignored)

For instance, we use RMS provided by J2ME to achieve the permanent information storage. We convert ports in the architecture to the methods enclosed by Java interface. And we implement various agents into a tree of generalized hierarchical agent classes.

\section{CONCLUSION}

We extend the design space by splitting it into function design space, architecture design space and system design space, which is the core idea of SADPBA, an 
architecture-centered design process. We develop MEECS, a system of mobile e-commerce, in the design phase of which we use SADPBA. In this case, three design phases are listed in more detail to illustrate how an element of one design space is mapped.

\section{REFERENCES}

[1] He and Jia, Zeng, "A Component Based Distributed Software Architectural Description Environment. "Mini-micro Systems pp.1637-1640, 2003.

[2] He and Jia "Model of Software Analysis and Design Process Based on Architecture". Journal of Xi'an Jiao tong University pp.591-594, 2004.

[3] $\mathrm{He}$ and Jia., "E-Commerce Oriented Knowledge Description Language". Journal of Chinese/information Proceedings, pp. 37-42, 2004.

[4] He Jia. \& Qin" Modeling and Checking the Behavior of Software Architecture". Journal of Computer Research and Development pp. $2018-2024,2005$

[5] Jia, X.," A Distributed Software Architecture Design Framework Based on Attributed Grammar". Journal of Zhejiang University Science,pp. 513-518,2005

[6] Wang, Zeng, He and Jia. "An Effective Language Film Facilitating Interface Representation under Limited Mobile Environment." Proceedings of the 7th International Conference for Young Computer Scientists.2003.

[7] Thomas G. Lane. "A Design Space and Design Rules for User Interface Software Architecture". Technical Report CMU/SEI-90-TR-22, Carnegie Mellon University Software Engineering Institute, October 1990.

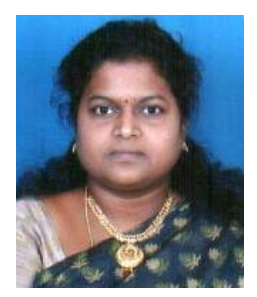

Dr. D.Vasumathi received the B. Tech. from JNT University, Hyderabad, M. Tech degree in Computer Science and Engineering from JNT University, Hyderabad and Ph. D in Computer Science and Engineering JNT University Hyderabad Andhra Pradesh, India. He worked as Assistant Professor, Presently working as Associate Professor in JNT University, Hyderabad .Shee has 12 years of Teaching experience. Currently guiding $8 \mathrm{Ph}$. D scholars. He is life member of ISTE and IE. Her research interests Data
Mining, Imageprocessing,Webtechnologies $\mathrm{He}$ has 5 international publications and 10 international conference Publications at International and National level.

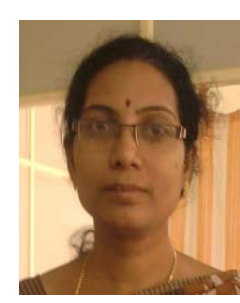

P. Rajarajeswari received the B. Tech. from S.V University, Sri Venkateswara University, Tirupathi, Andhra Pradesh.M. Tech degree in Computer Science from JNT University, Hyderabad and Pursuing Ph.D in Computer Science and Engineering, JNT University Hyderabad Andhra Pradesh, India. Presently She is working as Assistant Professor of Computer Science and Engineering in Madanapalle Institute of Technology and Science,Madanapalle,Chitoor(Dt),Andrapradesh. He has 8 years of and Teaching experiences. She is a life member of IAENGand IACSIT. Her research interests include Software Architecture, Software Engineering and Data Mining. He has 5international publications and 4 international conference Publications at International and 3National level workshops.

Dr. A. Rama Mohan Reddy received the B. Tech. from JNT University, Hyderabad in 1986, M. Tech degree in Computer Science from National Institute of Technology in 2000 Warangal and Ph. D in Computer Science and Engineering in 2008 from Sri Venkateswara University, Tirupathi, Andhra Pradesh, India. He worked as Assistant Professor, Associate Professor and Presently working as Professor of Computer Science and Engineering, Sri Venkateswara University College of Engineering. He has 28 years of Industry and Teaching experience. Currently guiding twelve Ph. D scholars. He is life member of ISTE and IE. His research interests include Software Architecture, Software Engineering and Data Mining. He has 10 international publications and 14 international conference Publications at International and National level. 